\title{
Desarrollo de pensamiento estratégico en la escuela de negocios
}

\author{
Miguel Ángel Corona \\ Jiménez \\ Universidad Iberoamericana \\ de Puebla \\ miguelangel.corona@iberopuebla. \\ edu.mx
}

\section{Resumen}

En ambientes de creciente competencia e incertidumbre por la innovación, característica rampante de la globalización, el uso de la inteligencia y la estrategia se ha vuelto casi indispensable; pensar estratégicamente es crucial porque ante la vorágine se requiere, en primer lugar, de una visión de futuro y, en segundo lugar, de pensar la forma en cómo llegar a ella considerando el comportamiento de los demás. En este trabajo se presentan los resultados de una experiencia al formar pensamiento estratégico en estudiantes del área de economía y negocios, mediante una metodología que integra la teoría como sustento básico del aprendizaje, su aplicación con una herramienta tecnológica que simula el ambiente de los negocios, cuyos resultados se comparan en el nivel internacional, complementada con una intervención en una organización, generalmente micro y pequeña, para diseñar su planteamiento estratégico. La aportación de este trabajo, además de la metodología, es la comprobación del avance en el pensamiento estratégico desde la óptica de los mismos sujetos de aprendizaje, los estudiantes, quienes a través de un instrumento de autoevaluación permiten identificar sus principales avances en términos de competencias: conocimientos, habilidades y actitudes para la dirección estratégica.

Palabras claves: pensamiento estratégico, evaluación-competencias, globalización y estrategia. 


\title{
Developing strategic thinking in a business school
}

\begin{abstract}
When the environment has an increasing competition and uncertainty for innovation, the application of intelligence and strategy is fundamental. Globalization is characterized by innovation, therefore strategic thinking is crucial. A vision of the future is required to analyze the business environment and then to make a decision which considers competitors. This research presents the results of students taking the course of strategic thinking. The students belonged to the faculty of Business and Economy. The methodology for the course was first teaching the text book theory, and then applying it with the use of a technological tool. This tool was a business environment simulation and the results were compared at international level. Finally, the students worked in teams to design the strategic planning of a local business. The main contribution of this research is the methodology and the evidence of the development of strategic thinking in the class. An instrument of evaluation was applied in the class, so the perspective of improvement comes from the student himself. The student identified his achievements and their growth rates are reported in terms of competences: knowledge, ability and attitude towards strategic management.
\end{abstract}

Keywords: strategic thinking, competence evaluation, globalization and strategy.

\section{Introducción}

En los últimos cincuenta años el mundo ha progresado tecnológicamente más que en todos los periodos anteriores. Después de la Segunda Guerra Mundial, muchos de los adelantos tecnológicos militares se incorporaron a la vida civil, principalmente en el área de los negocios; también muchos de los recursos económicos se destinaron a la investigación y al desarrollo, esencialmente en los países del norte, impulsando fuertemente el desarrollo de la microelectrónica, las telecomunicaciones y la computación, que sentaron las bases para apuntalar la evolución de las tendencias económicas y políticas que venían avanzando fuertemente hacia la globalización (Thurow,1992).

Con esos adelantos las nociones de tiempo y espacio cambiaron; la posible globalización de las actividades productivas y de las finanzas, así como la aceleración de las operaciones comerciales en la década de los ochenta comenzaron a presionar la apertura de las economías, que encontró en los planteamientos ultraliberales el "canto de las sirenas" que anticipaba el predominio del sistema de mercado y la 
solución a los problemas generados en el pasado por estados intervencionistas y reguladores, y hasta los rezagos por la desigualdad entre personas y regiones (Suárez, 2001).

En el nivel de las compañías se estaba inaugurando una etapa de creciente competencia y de cambios vertiginosos provocados por ese afán de mantener y acrecentar su participación en los mercados (Drucker, 1999). De hecho, ellas eran las que estaban impulsando la nueva era de la globalización convirtiendo al mundo, las regiones, los sectores y los mercados en arena para la creciente competencia y en un juego donde los mejor preparados serían los ganadores. La creciente competencia estaba obligando a reaccionar a los agentes económicos y las reacciones a su vez estaban provocando más cambios; el riesgo y la incertidumbre aumentaban (Thurow, 1992).

Para los noventa, los cambios se aceleraron con la emergencia de las industrias de alta tecnología, las telecomunicaciones, la Internet y la nueva economía de la red; aunado a esto, los procesos de integración económica por bloques estaban modificando la geografía de los centros de poder económico y las hegemonías monetarias. Las formas de hacer negocios se modificaban aceleradamente: la creciente competencia obligaba a las empresas no sólo a reducir sus tamaños y reestructurarse, sino a diseñar estrategias desde una óptica global, que abandonaban paradigmas de la dirección anterior (Drucker, 1993). Hasta en los ambientes locales estaban entrando dos conceptos poco comprendidos en su significado, pero muy sentidos por lo que representaban y afectaban: globalización y competitividad, sinónimos de apertura de las economías, libre circulación de bienes, servicios y capitales, reducción de costos, velocidad en la producción, aumento de la productividad y de la calidad, así como desempleo (Suárez, 2001).

Durante los primeros años del presente siglo, las tendencias tecnológicas, económicas, demográficas, políticas, sociales y ambientales se han acentuado. La tecnología ha permitido aumentar la productividad como nunca se imaginó Tomás Roberto Malthus; ${ }^{1} \sin$ embargo las desigualdades entre las personas y las regiones se han ampliado. Aunque ha continuado el crecimiento de las economías del norte, se percibe una notable preocupación por sus límites en cuanto a consumo de energía

\footnotetext{
${ }^{1}$ Estudioso de los procesos económicos y demográficos; sostuvo que la producción de alimentos crecía aritméticamente y la población geométricamente, lo que implicaba una visión pesimista del futuro para su época. R. Malthus (1951). Ensayo sobre el principio de la población. México: Fondo de Cultura Económica.
} 
y calentamiento global, pues la actual crisis ha puesto al descubierto que son necesarias principalmente mayores regulaciones financieras para los mercados. Las compañías se mueven en una incertidumbre continua provocada por todas aquellas que están innovando para mejorar su posición en el mercado; es una carrera que parece no tener fin, ni ganadores absolutos a primera vista; pero la necesidad de administrar el riesgo se vuelve un imperativo en un mundo en constante cambio. No se puede adivinar el futuro, mas sí imaginárselo y trabajar hacia él mediante estrategias que tengan como premisa una concepción sistémica de los negocios y su entorno, pero sobre todo que incorporen lo complejo de la realidad para tener más claridad y precisión en sus acciones. Se trata de pensar y actuar estratégicamente conforme se avanza.

Se observa que los que progresan son los que entienden que las nuevas condiciones de competencia demandan de gran conocimiento del entorno, de sus tendencias, de sus competidores, de valorar sus atributos y debilidades, pero, sobre todo, de visualizar el futuro, de fijar objetivos de largo plazo y hacer converger todos los esfuerzos para aprovechar las oportunidades; en otras palabras, de tener un pensamiento y una acción estratégica para ser competitivo.

En este sentido, si se quiere que México avance en su posición competitiva, además de que logre un desarrollo social y económico más equilibrado, es necesario que la gente no sólo se prepare científica, disciplinada o técnicamente, sino también que desarrolle un pensamiento y una praxis estratégicos que le permita visualizar el futuro, asignar mejor sus recursos, tomar decisiones considerando los cambios del entorno y concentrar sus esfuerzos con objetivos bien definidos en el largo plazo.

Lamentablemente, estos ejercicios de prospectiva se realizan con más frecuencia en las grandes compañías y en donde existe una administración y dirección profesionalizada; sin embargo, es necesario realizarlos en empresas de todos los sectores y de todos los tamaños, quizá más en las micros y pequeñas por la gran importancia que tienen en la generación de empleos. Los encargados de esas empresas deberían estar preparados en estos procesos o al menos quienes les pueden auxiliar; es decir, los egresados de las escuelas de negocios, así como de las universidades y tecnológicos en general.

En el caso de las escuelas de negocios, formar pensamiento estratégico para ser más competitivos es un imperativo que debe ser asumido con toda seriedad. No se trata de repetir el libro o manual de estrategia, ni de enseñar a analizar o aplicar 
técnicas (Mintzberg, 2004), sino de generar procesos de cambio en las personas a través de integrar conocimientos, habilidades y actitudes que les lleven a tomar decisiones considerando en todo momento objetivos de largo plazo, cambios continuos por la competencia y una ética de responsabilidad social. No es una tarea sencilla porque debe considerarse la complejidad del entorno y de la organización (Mintzberg, 2004); asimismo, es necesario asumir que las organizaciones son sistemas y a su vez subsistemas inmersos en un sistema más grande que el entorno, el cual puede ser sectorial, regional, nacional y mundial; todo esto para traducirlo en planteamientos estratégicos que posibiliten la competitividad de las organizaciones. Por lo tanto, no es una labor fácil y se deben utilizar los recursos tecnológicos pertinentes.

En este trabajo se presentan los resultados de formar pensamiento estratégico en estudiantes de administración de empresas, economía, contaduría y mercadotecnia de los últimos semestres ${ }^{2}$ en la Universidad Iberoamericana, Puebla, durante el periodo de primavera 2010. Esto desde una perspectiva integradora e incorporando los elementos teóricos necesarios, la utilización de tecnología a través de un simulador de negocios y el desarrollo de un planteamiento estratégico en una organización real.

\section{Pensamiento estratégico}

Pensar estratégicamente implica un proceso de visualizar el futuro, sembrarle objetivos y tender los puentes desde el presente considerando los cambios continuos del entorno. Parece sencillo de entender, pero requiere tener claro los conceptos que lo conforman; por un lado, lo que es el pensamiento y, por otro, lo que implica lo estratégico.

En esta lógica, primero definimos qué es el pensamiento para incorporar posteriormente lo estratégico. De acuerdo con Ángel Villarini Jusino, como todos los procesos humanos, el pensamiento es una actividad derivada de la generación de ideas sobre situaciones reales e imaginarias; además, es la capacidad humana para construir mentalmente representaciones e interpretaciones significativas de la relación con el mundo (Longoria et al., 2006). Por lo tanto, esta capacidad se desarrolla socialmente y es vital para adaptarse al medio ambiente tanto natural como histórico-cultural.

\footnotetext{
${ }^{2}$ Son los estudiantes que toman el curso de estrategia.
} 
Siguiendo con las ideas de Villarini, el sentido de las operaciones del pensamiento está definido por la necesidad que se tenga y por el objetivo propuesto. Para cumplir con su función, el sistema del pensamiento se conforma de tres subsistemas estrechamente relacionados:

1. El sistema de representaciones o codificación, que organiza los estímulos y la información de manera significativa, comprende imágenes, conceptos, nociones, esquemas.

2. El sistema de operaciones, que se encarga de procesar y reorganizar la información, comprende las destrezas intelectuales, las estrategias y tácticas del pensamiento, las heurísticas, los algoritmos, los métodos y las operaciones abstractas.

3. El sistema de actitudes, que trabaja con afectos que dan sentido y energía al pensamiento, comprende las emociones, los intereses, los sentimientos, los valores, y demás.

Estos elementos son los que van a permitir promover y generar actividades del pensamiento.

Ahora bien, el pensamiento estratégico es un proceso de razonamiento que utiliza el enfoque sistémico para entender situaciones complejas, donde la existencia de interrelaciones entre los variados elementos puede dar diferentes resultados a menos que se actúe con un plan para alcanzar determinados objetivos. Dicho pensamiento se considera como un recurso para administrar el riesgo disminuyendo la incertidumbre, descubriendo y aprovechando las oportunidades, utilizando el análisis y los aprendizajes (Loehle, 1996; Wells, 1998). Este enfoque surge a partir de considerar las condiciones del entorno y objetivos de largo alcance; es la base para la planeación estratégica y es de naturaleza intuitiva porque considera la visión, la misión, los valores y la estrategia (Morrisey, 1997). Además, el pensamiento estratégico soporta la coherencia del directivo y de la organización en la consecución de sus objetivos de largo plazo, pues se basa en acuerdos con quienes comparten el mismo pensamiento; aunque es intuitivo al plantear lo deseable de la empresa, es más analítico conforme avanza hacia la estrategia que requiere también del sentimiento y del análisis para sustentar las decisiones (Mintzberg, 1999).

Pensar estratégicamente requiere separar el todo en cada una de sus partes y luego juntarlas con los ajustes correspondientes para conformar el modelo que permita alcanzar los objetivos (Mintzberg, 2004); asimismo, implica flexibilidad intelec- 
tual para considerar situaciones cambiantes, distinguiendo en la coyuntura cada elemento y luego plantear su reestructuración para aprovechar oportunidades (Ohmae, 1996).

Desde una perspectiva más dinámica, el pensamiento estratégico conlleva una actitud proactiva para anticiparse al futuro; pensar en un futuro deseable, que no llegará solo y que requiere de tender puentes entre el presente y lo que se pretende mediante estrategias (Hamel y Prahalad, 1995); considerar que los recursos son escasos y que se deben administrar intertemporalmente; conocer el entorno y estar atentos a los movimientos de los competidores (Wootton \& Horne, 2003); identificar las tendencias relevantes del sector y del negocio; reconocer que la teoría del negocio envejece rápidamente y que es necesario estarse readaptando a los cambios continuos de la tecnología, de la sociedad y de las fuerzas económicas (Drucker, 1996).

Desarrollar pensamiento estratégico requiere ampliar los horizontes de la mente y trabajar los tres subsistemas del pensamiento; esto es, los procesos relacionados con los conceptos, conocimientos y esquemas, con las destrezas y habilidades mentales para plantear estrategias y utilizar métodos y técnicas que, finalmente con las emociones, motivaciones e intereses, moverán las voluntades.

En los últimos quince años, los enfoques de la planeación y la administración estratégica se han trasladado también hacia el campo del aprendizaje; desde esta premisa el pensamiento estratégico estaría apostando por desarrollar ventajas competitivas a partir de las organizaciones que aprenden (Senge, 1993; Mintzberg, 1999). Esto es lo que anima la iniciativa de formar pensamiento estratégico en jóvenes universitarios.

Si lo que se pretende es desarrollar pensamiento estratégico en jóvenes universitarios, será necesario incorporar en su formación estratégica al menos tres dimensiones: la adquisición y generación de conocimientos; el desarrollo de habilidades para procesar información y tomar decisiones; y la promoción de actitudes $\mathrm{y}$ valores para trabajar en equipo, respetar al otro y darle rectitud a sus objetivos estratégicos y financieros. 


\section{Modelo de formación de pensamiento estratégico}

Se plantea la formación de pensamiento estratégico considerando el funcionamiento de los tres sistemas que conforman el pensamiento: el sistema de representaciones, el de operaciones y el de actitudes, a los que se incorpora una concepción compleja de la realidad y el enfoque de sistemas para considerar efectos multifactoriales simultáneos, interrelaciones entre todos los elementos internos de la organización y con el entorno, así como las relaciones causa-efecto. La base para dar viabilidad a la conformación de representaciones y generación de conocimientos son la teoría, su aplicación en ejemplos y el ejercicio en la organización. Para dar cuenta de la complejidad, de las interrelaciones entre las variables y sus causalidades, el sistema de operaciones es estimulado por un conjunto de decisiones simultáneas y dinámicas de una organización en competencia a través del simulador de negocios.

Finalmente, complementando y equilibrando el desarrollo del pensamiento estratégico, el sistema de actitudes es activado y promovido por la cooperación del trabajo en equipo, considerando una ética de responsabilidad social en la toma de decisiones, a partir del juego de negocios (simulador) y de la interacción con el directivo y encargados de la organización para la elaboración del planteamiento estratégico.

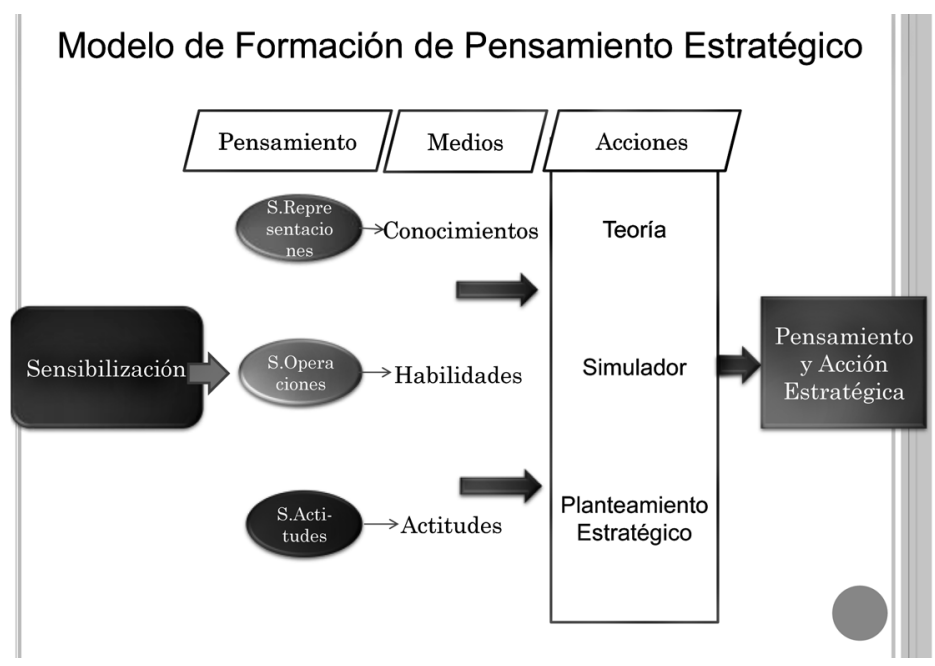

Desde el enfoque de competencias, la formación del pensamiento estratégico está en función de desarrollar conocimientos, habilidades y actitudes que conduzcan 
a un manejo adecuado del simulador y a la elaboración de un planteamiento estratégico, que permita a los sujetos de aprendizaje plasmar la complejidad de la realidad de los negocios, identificar las variables claves y sus interrelaciones y, lo más importante, desarrollar los procesos humanos suficientes para trabajar en conjunto, desarrollando competencias al menos en las siguientes dimensiones: comunicación, liderazgo intelectual, organización de personas y tareas, innovación y cambio, manejo de sí mismo, toma de decisiones en ambientes de competencia y perspectiva global con ética de responsabilidad social.

\section{Metodología para desarrollar pensamiento estratégico ${ }^{3}$}

Lo primero que debe incorporar una metodología para desarrollar pensamiento estratégico es una declaración sobre la importancia del futuro, de fijar objetivos a largo plazo, de que el entorno cambia constantemente y, por lo tanto, se está condenado a readaptarse continuamente so pena de quedar marginado por la competencia.

Segundo, se trata de una formación rigurosa que parte de bases teóricas sólidas y profundas, que rebasan en mucho las formaciones light que se dan sólo con herramientas (Mintzberg 2004). Esta propuesta comienza por una noción de lo complejo de la realidad y de la importancia de utilizar el enfoque de sistemas; en esta parte es fundamental la contextualización que descubre las interrelaciones entre los diferentes elementos de la organización y con el entorno económico, social, cultural y natural. Incorpora también la adquisición de conocimientos y el procesamiento de la información mediante métodos y técnicas cuantitativas y cualitativas para situaciones reales; esto se lleva a cabo con trabajo en el aula y lecturas fuera de ella.

Tercero, la apreciación de lo complejo y sistémico se hace a partir de una experiencia vivencial, participando en un simulador de negocios, ${ }^{4}$ ésta es una herramienta tecnológica muy importante que permite al estudiante visualizar las partes de una organización interactuando con un entorno global sumamente competitivo y dinámico, que lo lleva a trabajar en equipo para tomar decisiones en cada una de

\footnotetext{
${ }^{3}$ La metodología que se presenta se ha aplicado durante seis semestres en el curso de estrategia que se ofrece a estudiantes de economía y negocios en la Universidad Iberoamericana de Puebla, periodo durante el cual se ha ido innovando para mejorar sus resultados.

${ }^{4}$ Mayor información en: http://www.bsg-online.com.
} 
las áreas de la organización. El alumno requiere utilizar todos los conocimientos previos de su carrera más los aprendidos en el presente curso de estrategia. Es una suma de ejercicios de integración y aplicación de conocimientos y habilidades, cohesionados por actitudes e intereses de cooperación, de generación de valor y de competitividad. También requiere trabajo con la computadora, dentro y fuera del aula, los resultados del juego se revisan después de cada jugada y se reconoce si aparecen los equipos entre los mejores en el mundo, según las comparaciones que hacen los administradores del simulador de negocios en los Estados Unidos.

Cuarto, el desarrollo de habilidades de diagnóstico, de propuesta y de ejercicio de valores se realiza a partir de la elaboración de un planteamiento estratégico para una organización real, lo que implica trabajar continuamente con el empresario o director, ofrecerle ventajas y alternativas, y ser calificado en presentación final por el propio beneficiario. Esto implica trabajo fuera del aula con asesoría continua del profesor.

Quinto, la forma en que se administra el curso es mediante la plataforma Moddle, la evaluación de todas las actividades del curso se hace con exámenes para la parte teórica, con los resultados del simulador y con el trabajo de la empresa. No obstante, la mejor manera de saber si hubo cambios en la manera de pensar, en el desarrollo de un pensamiento estratégico, es a través de una evaluación de proceso de cómo llegó el estudiante y de cómo salió. Para identificar esto el único que lo sabe y percibe es el propio sujeto de aprendizaje; por lo tanto, es necesario un ejercicio de autoevaluación que requiere de un clima de confianza que debe construirse a lo largo de todo el curso. Los resultados de la aplicación de este instrumento de autoevaluación son los que se reportan en este trabajo.

Sexto, el instrumento de autoevaluación es un cuestionario que pregunta sobre el desarrollo o avance durante el curso sobre conocimientos, habilidades y actitudes relacionadas con el objetivo de desarrollar pensamiento estratégico en sus tres dimensiones: el saber, el saber hacer y el ser. La escala de evaluación es del 1 al 5, tanto para calificar cómo llegaron y cómo salieron del curso en cada ítem. De esta manera es posible calcular tasas de crecimiento e identificar las dimensiones más desarrolladas del pensamiento estratégico en acción.

A continuación se presentan los resultados del proceso de desarrollo de pensamiento estratégico en dos grupos de estudiantes que llevaron el curso de estrategia en el semestre de primavera 2010. El primero de ellos estuvo formado por 14 es- 
tudiantes de administración de empresas, en tanto que el segundo fue mixto: 10 de economía, 2 de mercadotecnia y 1 de contaduría.

\section{Resultados del proceso de desarrollo del pensamiento estratégico}

Para entender mejor esta parte es conveniente sentar un antecedente; esto es, aclarar que al inicio del curso se hace en una sesión de diagnóstico para reconocer los conocimientos previos y las habilidades adquiridas sobre la dirección de negocios y su enfoque estratégico. Se observa en la mayoría de los estudiantes que su conocimiento sobre lo que significa lo estratégico es ambiguo, confunden generalmente acciones operativas con estrategias, se les dificulta pensar en el futuro y concebir que la realidad, y en especial la de los negocios, es cambiante. También no comprenden que las decisiones que ellos tomen van a afectar a todas las áreas de la organización en un efecto dominó; conciben a la organización como un ensamble de partes, pero no precisamente como un sistema circulatorio y casuístico, quizá porque han aprendido conocimientos de manera fragmentada en materias aisladas sin articulación. Precisamente, este curso por su objetivo es integrador y permite evaluar holísticamente el proceso en términos de competencias.

Después del diagnóstico, sigue la etapa de sensibilización donde se les plantea la disyuntiva de ser competitivos o quedar marginados por el mercado. Se utilizan ejemplos de los negocios y de la vida cotidiana. Con esto se da paso a la parte teórica que contiene todo lo necesario para desarrollar un planteamiento estratégico ${ }^{5}$, cada parte se ejemplifica, pero sobre todo se aplica en el trabajo de intervención en una organización que se realiza en equipos de cuatro personas.

El trabajo de intervención comienza en la segunda semana del curso. ${ }^{6}$ Cada semana se asesora a los estudiantes para abordar la relación y el trabajo con el empresario o director. Se les pide dos presentaciones de avances con evidencias que se evalúan. En la presentación final asiste el beneficiado que calificará el trabajo y discutirá con el grupo los resultados; en el formato de evaluación se pregunta sobre el des-

\footnotetext{
${ }^{5}$ Componentes del planteamiento estratégico: visión, misión, filosofía, valores, cultura organizacional, objetivos estratégicos y financieros, análisis del macroambiente, análisis del sector de competencia, fuerzas impulsoras del cambio, análisis de grupos estratégicos, FODA, cadena de valor y su costeo, benchmarking, factores determinantes del sector, práctica ético-valoral, ventaja competitiva y distintiva, además de estrategias competitivas.

${ }^{6} \mathrm{El}$ curso se desarrolla en 16 semanas.
} 
empeño del equipo, su conocimiento del tema, la organización y responsabilidad que demostraron, la forma cómo se relacionaron para trabajar, el planteamiento estratégico; en especial hay dos preguntas cruciales: 1) ¿Si el trabajo presentado le muestra una clara diferencia entre lo que tenía y lo que le proponen? y 2) ¿Si estaría dispuesto a contratar los servicios del equipo que le asesoró? En esta parte los cambios en los estudiantes son evidentes, la mayoría utiliza de manera fluida el lenguaje estratégico, identifica con precisión lo que significa estratégico, en sus presentaciones se nota consistencia entre los análisis y las propuestas; cabe destacar que también es posible observar el trabajo en equipo. Es de reconocer que en todos los casos el empresario o directivo ha mostrado satisfacción sobre el trabajo de los muchachos y con el planteamiento estratégico.

La otra parte crucial es la del manejo del simulador de negocios que inicia en la tercera semana del curso con la lectura del manual del jugador que está en inglés, el cual aunque es extenso y complejo, no resulta difícil; es la primera prueba que requiere del enfoque sistémico, pues el manual va explicando cada uno de los elementos y sus posibles interrelaciones; asimismo, es la prueba para los conocimientos adquiridos previamente; cabe señalar que si los alumnos no llevaron bien sus cursos de administración, economía, contabilidad y finanzas, mercadotecnia, personal y calidad, se les dificultará el manejo de la empresa y la toma de decisiones. Afortunadamente, el simulador tiene dos jugadas de ensayo que son una llamada de atención para aquellos que pretenden competir sin estar preparados. En la jugada 14 se requiere elaborar un plan estratégico para garantizar la utilización del herramental teórico y tecnológico. El comportamiento de los estudiantes es muy sui generis; se puede observar que se encuentran los que leyeron sin cuidado el manual y los cuidadosos; además de los preocupados porque el $30 \%$ de la calificación del curso será el resultado que obtengan y los que además quieren aprender.

Los productos al final de la jugada 20 fueron muy significativos, no tanto por los índices alcanzados, sino por lo que declararon los participantes en la presentación de sus resultados. En un ejercicio de absoluta transparencia descubrieron las estrategias que ejecutaron, los errores que cometieron y los aprendizajes que tuvieron. Fue una sesión de aprendizaje y reconocimiento al esfuerzo, por lo que se felicitó a los que salieron en la lista de los cien mejores del mundo, que fueron dos. ¿Qué fue lo valioso de esta parte? Los participantes reconocieron lo importante que es tener objetivos de largo plazo y planear la estrategia para alcanzarlos; que todas las partes de la empresa son muy importantes porque interactúan; que toda decisión implica costos y riesgos; que no se deben tomar decisiones sin analizar el mercado 
y las reacciones de la competencia; asimismo, que las decisiones en cada empresa van moldeando el perfil de competencia de la industria, entre otros aspectos.

Con los exámenes escritos que incluyeron lo teórico, el trabajo de intervención en la empresa y los resultados del simulador se integró la calificación final del curso; con ello se puede demostrar cómo se desarrolla el pensamiento estratégico, así como qué tanto se avanzó en términos de conocimientos, habilidades y actitudes reconocidas por el propio estudiante. A continuación se explican estos resultados.

\section{Autoevaluación y percepción}

Para conocer los avances en el pensamiento estratégico desde la óptica de los estudiantes se realizó un ejercicio de autoevaluación, que requirió previamente de generar un clima de confianza y de sinceridad. Para garantizar que sus respuestas no afectaran la evaluación final, se aplicó el instrumento justo antes de entregar las calificaciones finales y se explicó a los estudiantes la intención e importancia de su participación. El instrumento estuvo integrado por tres secciones: conocimientos, habilidades y actitudes, cada una relacionada con un sistema de la estructura del pensamiento: de representaciones, de operaciones y de actitudes. A su vez cada sección estuvo compuesta por siete ítems o aspectos del pensamiento estratégico, que fueron evaluados con una escala ascendente del 1 al 5 considerando dos momentos en el tiempo, cuando iniciaron y cuando terminaron el curso.

Al ser una autoevaluación referida al proceso, el estudiante sabía mejor cómo se encontraba con respecto a ese ítem al comenzar el curso y cómo se encontró al final para asentar las calificaciones respectivas. Con sus respuestas era posible identificar diferencias positivas o negativas y calcular esos cambios con tasas de crecimiento o decrecimiento. Es así que el estudiante podía conocer sus mayores avances en competencias, tanto a nivel agregado como de ítem. Los resultados que se procesaron fueron los valores promedio de cada ítem calculados a partir de las respuestas individuales. Hay que recordar que se trata de dos grupos, el primero integrado por estudiantes de administración de empresas y el otro mixto integrado en su mayoría por alumnos de economía, acompañados por estudiantes de mercadotecnia y de contaduría. Esta connotación es importante por las diferencias que se encontraron. 


\section{Resultados en el nivel agregado}

En primer lugar es importante señalar que en todos los ítems tanto en el grupo de administradores como en el mixto hubo diferencias positivas, lo que indica que hubo avances en la adquisición de conocimientos, desarrollo de habilidades y mejora o reforzamiento de actitudes. Ahora bien, de acuerdo con las gráficas 1 y 2 , que aparecen más adelante, los avances no fueron homogéneos: en conocimientos, el grupo mixto manifestó un cambio mayor de $66.1 \%$ en comparación con el grupo de los administradores que fue de 55.8\%; posiblemente, la diferencia se debió a que el grupo mixto tenía menos antecedentes relacionados con el curso debido al contenido temático de sus carreras, por lo que aprendieron más conocimientos nuevos. En cuanto al desarrollo de habilidades, el grupo de los administradores mostró mayores avances con $64.5 \%$ frente a $55.7 \%$ del grupo mixto; esta diferencia puede deberse a que para los administradores era menos familiar el uso de herramientas de análisis tanto cuantitativas como cualitativas. Los menores avances, pero no por ello menos significativos, los registraron ambos grupos en las actitudes aunque con diferencias. Los administradores reconocieron un avance del $25.7 \%$ en comparación con el 13.0\% de los que formaron el grupo mixto; esta diferencia de casi el doble puede estar explicada por el mayor acercamiento de lo social en el caso de los estudiantes de economía; otra explicación obligada es la que se refiere al menor avance de lo actitudinal frente a los conocimientos y las habilidades, al respecto puede argumentarse que al ser la Universidad Iberoamericana de orientación humanista, la formación ética-valoral se da desde los primeros semestres, por lo que es muy posible que después del cuarto semestre los cambios en este aspecto sean menores, prueba de ello son los valores promedio registrados por los estudiantes al inicio del proceso, que son altos.

\section{Resultados en el nivel desagregado}

Analizando los ítems relacionados con los conocimientos, los avances más reconocidos en el grupo de los administradores fueron los relacionados con las estrategias para competir, con el entorno de los negocios, con la utilidad del benchmarking y con el enfoque sistémico. En el grupo mixto fueron: planear las actividades, desarrollar una visión de largo plazo y las estrategias para competir, así como el enfoque sistémico. Como se mencionó anteriormente, los crecimientos en este último grupo fueron superiores, lo cual se puede apreciar en el cuadro 1, que aparece más adelante. 


\section{Gráfica 1}

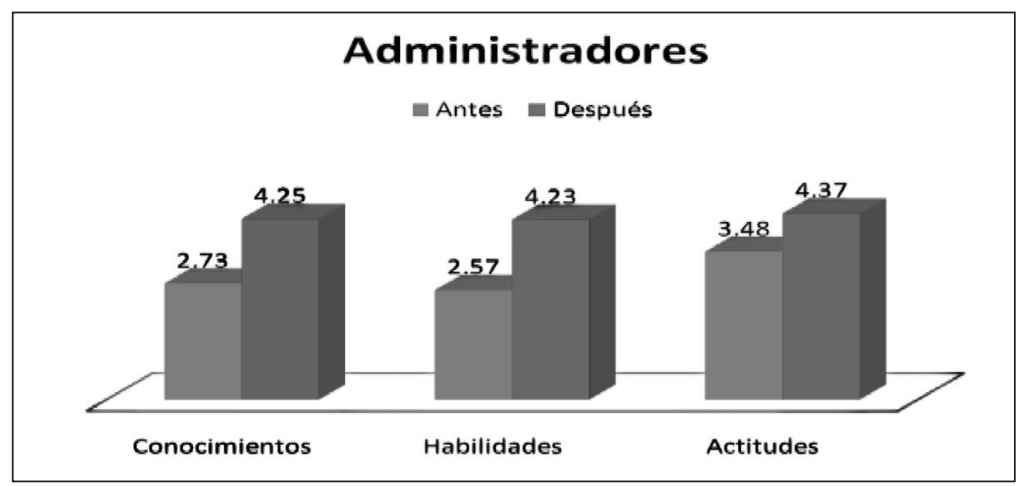

Fuente: Elaboración propia con base en la autoevaluación de estudiantes sobre desarrollo del pensamiento estratégico. Primavera de 2010.

\section{Gráfica 2}

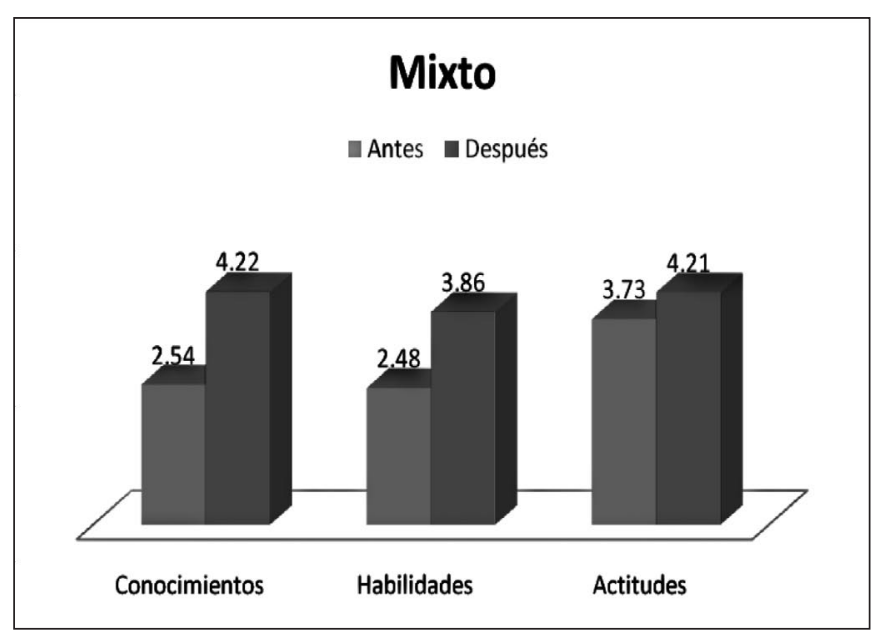

Fuente: Elaboración propia con base en la autoevaluación de estudiantes sobre desarrollo del pensamiento estratégico. Primavera de 2010.

Por lo que respecta a los ítems relacionados con el desarrollo de habilidades, tanto en el grupo de los administradores como en el mixto, los dos que mayor crecimiento tuvieron fueron: el manejo del Diamante de Porter y la elaboración de mapas de 
grupos estratégicos. Siguieron en crecimiento para los administradores el manejo de objetivos estratégicos y financieros y la elaboración de la cadena de valor; en tanto que para los integrantes del grupo mixto fueron el desarrollo de ventaja competitiva y también la elaboración de la cadena de valor.

Finalmente, por lo que respecta al análisis de los ítems de la sección de actitudes, los crecimientos fueron un tanto diferentes para ambos grupos. Para los administradores, la observación de una ética de responsabilidad social en la toma de decisiones resultó la de mayor crecimiento, siguieron la disciplina y responsabilidad en el curso, la valoración de las personas y la disposición para trabajar con el empresario o directivo. Para el grupo mixto, esta última resultó ser la de mayor avance, siguiendo la de observación de una ética de responsabilidad social en la toma de decisiones, el ejercicio de la disciplina y responsabilidad en el grupo, así como la promoción de la justicia y la equidad a través de las acciones. Aunque en ambos grupos se notaron crecimientos en todos los ítems, los mayores fueron reconocidos por los administradores.

En general se puede afirmar que se lograron avances importantes en el desarrollo de un pensamiento estratégico, percibidos y reconocidos por el propio sujeto del aprendizaje, lo cual se complementa y constata con lo mencionado anteriormente en la evaluación hecha por el empresario sobre la elaboración del planteamiento estratégico y con los resultados alcanzados en el simulador de negocios. 


\section{Cuadro 1}

Autoevaluación, tasas de crecimiento de los valores promedio por ítems, agrupados por conocimientos, habilidades y actitudes

\begin{tabular}{|c|c|c|c|c|}
\hline ITEMS & & Administradores & & Mixto \\
\hline & 58.06 & $\%$ & & $\%$ \\
\hline Conocimientos & & 55.81 & & 66.10 \\
\hline Enfoque sistémico de los negocios & & & & 65.52 \\
\hline $\begin{array}{l}\text { Importancia de una visión estratégica de } \\
\text { largo plazo }\end{array}$ & & 48.84 & & 96.43 \\
\hline Estrategias para competir en los negocios & & 73.53 & 57.14 & 86.21 \\
\hline Entorno de los negocios & & 68.57 & & 30.77 \\
\hline Importancia de generar valor principal & & & & 59.38 \\
\hline $\begin{array}{l}\text { Utilidad del } \\
\text { Benchmarking }\end{array}$ & & 58.54 & & 113.04 \\
\hline Planear las actividades antes de realizarlas & & 34.78 & & 40 \\
\hline Habilidades & 108.00 & 64.52 & & 55.71 \\
\hline Manejo de objetivos estratégicos y financieros & & 84.38 & & 38.89 \\
\hline Manejo del diamante de Porter & & 68.57 & & 145.00 \\
\hline Manejo del FODA & & 36.17 & & 36.84 \\
\hline Elaboración de la cadena de valor & & & & 92.00 \\
\hline Desarrollo de ventaja competitiva y distintiva & & 55.26 & & 103.85 \\
\hline Elaboración de mapas de grupos estratégicos & & 87.50 & & 122.73 \\
\hline Diálogo para organizar y tomar decisiones & & 44.19 & & 14.63 \\
\hline & 24.49 & & & \\
\hline Actitudes & 27.45 & 25.70 & & 13.03 \\
\hline Cumplir con los compromisos en equipo & & 8.33 & & 8.33 \\
\hline Trabajar con el directivo, empresario & & & & 32.43 \\
\hline $\begin{array}{l}\text { Considerar lo valioso de las personas } \\
\text { en la organización }\end{array}$ & & 27.66 & & 15.91 \\
\hline Promover la justicia y la equidad con las acciones & & 16.00 & 11.11 & 11.36 \\
\hline $\begin{array}{l}\text { Observancia de la ética de responsabilidad } \\
\text { social en la toma de decisiones }\end{array}$ & & 34.78 & & 8.51 \\
\hline $\begin{array}{l}\text { Ejercicio de la disciplina y la responsabilidad } \\
\text { en el curso }\end{array}$ & & 33.33 & & \\
\hline $\begin{array}{l}\text { Considerar el medio ambiente en la toma } \\
\text { de decisiones }\end{array}$ & & 20.00 & & 8.51 \\
\hline
\end{tabular}




\section{Conclusiones}

Si se pretende mejorar la competitividad en un país no basta con enfocarla sólo desde lo macro; las organizaciones y las personas son las que compiten, por lo que es necesario tener claridad sobre el concepto y la forma de cómo poder desarrollarla. Uno de los elementos claves para crecer en ambientes de competencia es la administración estratégica, que tiene teorías, métodos y técnicas, además de que se inicia por la forma de observar la realidad y los negocios, por ver más allá del corto plazo y por entender que el entorno cambia continuamente por las decisiones que toman los agentes participantes. Esto es lo que experimentan los que desarrollan un pensamiento estratégico.

Para desarrollar un pensamiento estratégico es necesario considerar cómo se estructura el pensamiento del ser humano a través de sus tres sistemas: representaciones, operaciones y actitudes para complementarlo con dos visiones o enfoques: el de la complejidad y el de lo sistémico. Considerando estos elementos se tienen que desarrollar actividades que vayan conformando la estructura del pensamiento a partir de conocimientos, habilidades y actitudes permeadas por lo estratégico.

A partir de lo presentado en este trabajo es posible concluir que la teoría contribuye a la construcción de conocimientos y significados, pero que es fundamental desarrollar actividades que confronten las ideas con la realidad que se observa y la que está más allá de nuestros alcances, la elaboración de un planteamiento estratégico para una organización es un gran medio para desarrollar habilidades del pensamiento estratégico, al igual que dirigir y operar una empresa en pleno movimiento competitivo, que se logra con el manejo del simulador de negocios. Con ambas herramientas es posible integrar conocimientos, desplegar habilidades para competir y ejercitar actitudes para relacionarse mejor con las personas y con el entorno. El trabajo en equipo, la actitud de mejorar, el respeto por las personas y por el medio ambiente tienen una oportunidad importante de aplicación.

El resultado de los procesos descritos desde la óptica de la autoevaluación de los estudiantes demostró que los mayores progresos fueron en conocimientos y en habilidades. La parte actitudinal fue la de menores avances, pero se explica porque gran parte de lo medido con el instrumento ya había sido desarrollado en etapas anteriores de la formación de los estudiantes, como lo demostró el alto valor promedio de los ítems al inicio del curso. 
Por último, el desarrollo del pensamiento estratégico no es sencillo, requiere de un ejercicio disciplinado, guiado por una metodología rigurosa como la que se presenta en este trabajo, donde la herramienta tecnológica tiene un papel clave. Lo reportado es el resultado de una cuidadosa planeación y ejecución que hizo converger todos los esfuerzos para lograr el objetivo de hacer pensar a los estudiantes estratégicamente; ésa es la mentalidad para ser competitivos.

\section{Referencias}

Drucker, P. (1993). Gerencia para el futuro. Bogotá: Grupo Editorial Norma.

(1996). Drucker, su visión sobre la administración, la organización basada en la información, la economía y la sociedad. Bogotá: Grupo Editorial Norma.

(1999). Los desafíos de la administración en el siglo XXI. Buenos Aires: Sudamericana.

Hamel, G. y C.K. Prahalad (1995). Compitiendo por el futuro, estrategia crucial para crear los mercados del mañana. Barcelona: Ariel Sociedad Económica.

Loehle, C. (1996). Thinking strategically: power tools for personal and professional advancements. New York: Cambridge University Press.

Longoria, R., I. H. Cantú y D. S. Ruiz (2006). Pensamiento creativo. México: Universidad Autónoma de Nuevo León, CECSA (Colección Estudios Generales).

Mintzberg, H. (1999). Gerencia estratégica. México: Mc Graw-Hill. (2004). Managers, not MBAs: a hard look at the soft practice of managing and management development. San Francisco: Berrett-Koehler Publishers Inc.

Morrisey, G. (1997). Pensamiento estratégico. México: Prentice Hall. 
Ohamae, K. (1996). La mente del estratega. México: Mc Graw-Hill.

Porter, M. (1987). Ventaja competitiva. Creación y sostenimiento de un desempeño superior. México: Compañía Editorial Continental.

Senge, P. (1993). La quinta disciplina. Buenos Aires: Granica.

Suárez Suárez, A. (2001). Nueva economía y nueva sociedad: Los grandes desafíos del siglo XXI. Madrid: Financial Times-Prentice Hall.

Thompson, A. y A. Strickland (2008). Administración estratégica, conceptos y casos. $15^{a}$ ed., México: Mc Graw-Hill

Thurow, L. La guerra del siglo XXI: La batalla económica que se avecina entre Japón, Europa y Estados Unidos. Buenos Aires: Vergara.

Villarini Jusino, A. (s.f.). Teoría y pedagogía del pensamiento sistemático y crítico, Universidad de Puerto Rico: Organización para el Fomento del Desarrollo del Pensamiento. Disponible en: http://encuentropensamiento.org/docs/Teo ria\%20y\%20pedagogia\%20del\%20pensamiento.PDF.

Wells, S. (1998). Choosing the future, the power of strategic thinking. Boston: Butterworth-Heinemann.

Wheelen, T. y D. Hunger (2007). Administración estratégica y política de negocios, conceptos y casos. 10a ed., México: Pearson-Prentice Hall.

Wootton, S. y T. Horne (2003). Strategic thinking, a step by step approach to strategy. 2nd ed., UK: Kogan Page Ltd. 